\title{
QUALIDADE DE VIDA: CONCEITO E VARIÁVEIS RELACIONADAS
}

Érico Felden Pereira

Anderlei dos Santos

Clarissa Stefani Teixeira

Lizandra Salau da Rocha

Rosimeide Francisco Santos Legnani

Eugenio Andrés Díaz Merino

\section{Resumo}

Devido à falta de um conceito amplamente aceito a qualidade de vida é tratada por diferentes óticas conforme a área e a situação de interesse, sendo, em muitos casos, considerada sinônimo de saúde. Assim, este artigo tem por objetivo realizar uma apresentação de algumas abordagens conceituais e discutir algumas variáveis relacionadas à qualidade de vida como saúde, estilo de vida, bem-estar, felicidade, liberdade, autonomia, possibilidades de crescimento e percepção. As leituras realizadas apontam para a necessidade do tema ser tratado de forma ampla e multidisciplinar e da investigação de estratégias claras para a promoção da qualidade de vida dos indivíduos.

\section{Palavras-Chave}

Qualidade de vida; Saúde e bem-estar

\section{CALIDAD DE LA VIDA: CONCEPTO Y LAS VARIABLES RELACIONADAS}

Érico Felden Pereira

Anderlei dos Santos

Clarissa Stefani Teixeira

Lizandra Salau da Rocha

Rosimeide Francisco Santos Legnani

Eugenio Andrés Díaz Merino

\section{Resumen}

Debido a la falta de un concepto completamente acepte la calidad de vida es tratada por ópticas diferentes según el área y la situación de interés, siendo, en muchos casos, considerado sinónimo de salud. Así, este artículo tiene como objetivo realizar una presentación de algunos acercamientos conceptuales y discutir algunas variables relacionadas a la calidad de vida como la salud, estilo de vida, bienestar, felicidad, libertad, autonomía, posibilidades de crecimiento y percepción. Las lecturas realizadas apuntan la necesidad del tema ser tratado sobre la forma ancha y multidisciplinar y de la investigación de las estrategias claras para la promoción de la calidad de la vida de los individuos.

Palabras-chave: calidad de la vida; salud y bienestar

\section{QUALTY OF LIFE: CONCEPT AND RELATED VARIABLES}

\begin{abstract}
Due to the lack of a concept largely accepted, the quality of life is treated by different points of view according to the area and situation of interest, and it is considered, in many cases, as synonymous of health. Thus, this article has as objective to make a presentation of some conceptual approaches and some
\end{abstract}


variables related to the quality of life as health, life style and happiness, freedom, autonomy and possibilities of growth and perception. The readings done point out the necessity of the theme to be treated in an ample and multidisciplinary and the invetigable clear strategies for the promotion of people's quality of life.

\section{Key-Words}

Quality of life; Health and welfare 


\section{INTRODUÇ̃̃O}

O tema qualidade de vida vem recebendo destaque em várias áreas de pesquisa e suas definições são tão numerosas e divergentes quanto os métodos de avaliação (FERRISS, 2006). A investigação sobre qualidade de vida ganha espaço quando se busca compreender e sugerir formas para que as pessoas possam viver melhor, bem como orientar políticas públicas e tratamentos de saúde que tratem o ser humano de forma mais ampliada (FARQUHAR, 1995). Apesar da falta de consenso sobre o conceito de qualidade de vida, três aspectos fundamentais referentes ao construto são apontados pela literatura: (1) subjetividade; (2) multidimensionalidade e (3) presença de dimensões positivas como mobilidade e negativas como a dor (WHOQOL, 1998).

De acordo com Tani (2002) qualidade de vida significa muito mais que ter atendidas as necessidades básicas de sobrevivência, como alimentação, vestuário, trabalho e moradia. Implica em ter saúde física e mental, relações sociais harmoniosas e construtivas, educação permanente, relacionamento respeitoso e amigável com o meio ambiente, tempo livre para o lazer e oportunidades para usufruir a cultura em sua plenitude. Em razão dessa multiplicidade de fatores, estudos e teorizações sobre qualidade de vida, ainda não conseguem estabelecer com clareza a parcela de contribuição desses aspectos, tampouco suas interações. O que pode ser entendido é apenas que qualidade de vida não é a simples soma dos fatores acima referidos, aplicando-se muito bem à sua compreensão aquele princípio sistêmico de que o todo é maior que a soma das partes.

Dependendo da área de interesse o conceito, muitas vezes, é adotado como sinônimo de saúde (MICHALOS; ZUMBO; HUBLEY, 2000; SCHMIDT et al., 2005), felicidade e satisfação pessoal (RENWICK e BROWN, 1996), condições de vida (BUSS, 2000), estilo de vida (NAHAS, 2003), dentre outros; e seus indicadores vão desde a renda até a satisfação com determinados aspectos da vida. Devido a essa complexidade, conforme abordam Seild e Zanonn (2004) e Dantas; Sawada; Malerbo (2003), qualidade de vida apresenta-se como uma temática de difícil compreensão e necessita de certas delimitações que possibilitem sua operacionalização em análises científicas.

Pires, Matiello; Gonçalves (1998) discutem que é possível perceber uma crescente, porém pouco esclarecedora aplicação do conceito de qualidade de vida. Objetivando uma facilidade de pesquisa e discussão, o tema em muitos casos, é reduzido em sua complexidade dogmatizando a expressão e criando dificuldades para o diálogo interdisciplinar necessário à sua compreensão. O uso indiscriminado do tema 
por várias áreas do conhecimento, somando à utilização cotidiana no discurso publicitário, faz com que qualidade de vida seja uma expressão que ao pretender dizer muito pode acabar nada esclarecendo.

Desta forma, diante da importância social, política e acadêmica do tema qualidade de vida e por esse ainda apresentar imprecisões conceituais, metodológicas e sobre quais variáveis podem ser investigadas quando se pretende avaliar a qualidade de vida, este artigo tem por objetivo realizar uma discussão conceitual e abordar as variáveis comumente apontadas nos estudos com qualidade de vida.

\section{CONCEITO}

A qualidade de vida é considerada como a percepção do indivíduo de sua posição na vida, no contexto da cultura e sistema de valores nos quais vive e em relação aos seus objetivos, expectativas, padrões e preocupações (WHOQOL, 1994). Recorrendo-se à etimologia do termo qualidade, ele deriva de qualis [latim] que significa o modo de ser característico de alguma coisa tanto considerado em si mesmo como relacionado a outro grupo, podendo assim assumir tanto características positivas como negativas. Porém, quando se fala em qualidade de vida, acredita-se que, geralmente, refere-se a algo bom, digno e positivo (SANTIN, 2002).

Tani (2002) discute ainda que, a exemplo da qualidade de vida, determinados aspectos da vida como a felicidade, amor e liberdade, mesmo expressando sentimentos e valores difíceis de serem compreendidos, não se tem dúvida quanto a sua relevância. Trata-se de um conceito para o qual até mesmo uma definição operacional é difícil de ser elaborada. Qualidade de vida vem sendo uma idéia largamente difundida na sociedade, correndo-se o risco de haver uma banalização do assunto pelo seu uso ambíguo, indiscriminado ou oportunista. Por um lado, se tem a exploração oportunista de um conceito o que resulta na sua depreciação e, de outro, o reconhecimento de que esse conceito exprime uma meta nobre a ser perseguida, resultando na preservação de seu significado e valor.

Segre e Ferraz (1997) abordam que dentro da bioética e considerando o conceito de autonomia, entendese que qualidade de vida seja algo intrínseco, só possível de ser avaliado pelo próprio sujeito, sendo difícil afirmar com clareza se há uma "boa" ou "má" qualidade de vida porque a realidade é a de cada um. Nessa discussão, se enfatiza a prioridade do subjetivismo em toda reflexão sobre qualidade de vida, considerando inclusive os parâmetros de "normalidade" impostos pela sociedade. Poderá alguém afirmar que um portador de colostomia, conseqüente a uma cirurgia de câncer intestinal, tem qualidade de vida pior do que um seguidor obsessivo de regras religiosas, intimidado perenemente por um Deus que the foi 
inculcado, independentemente de sua vontade? Nesta óptica, vai ficando claro que "realidade" nada mais é do que uma convergência de subjetivismos - tensão entre homem e sociedade.

Com base no estudo do conceito e das formas de avaliação do construto qualidade de vida verifica-se que este se consolida como uma variável importante na prática clinica e na produção de conhecimento na área da saúde e áreas afins. Mesmo com as inúmeras controvérsias sobre sua conceituação e estratégias de mensuração, os estudos até então realizados têm contribuído para a clarificação do conceito e sua relativa maturidade. Acredita-se que o estudo do tema pode contribuir para a criação de formas mais adequadas e humanas de atendimento à saúde e na consolidação de novos paradigmas do processo saúde-doença e das políticas públicas (SEIDL; ZANNON, 2004).

Os estudos publicados sobre o tema revelam uma tendência da investigação das condições de vida como um indicativo de qualidade de vida em diferentes grupos, em investigações relacionadas com patologias ou não, bem como dos estados de saúde ou estado funcional (GILL; FEINSTEIN, 1994; BUSS, 2000). Perceptível também uma preocupação com o local onde as pessoas vivem principalmente o meio urbano que muitas vezes torna-se insalubre devido à poluição ambiental, visual e sonora, transito mal organizado, poucos espaços disponíveis para o lazer dentre outros (FORATTINI, 1991; RENWICK e BROWN, 1996; NAHAS, 2003).

Outra tendência das publicações é investigar quais aspectos dentro da qualidade de vida como, por exemplo, condições sociais, físicas ou psicológicas são mais relevantes para análise geral (PEREIRA et al., 2006), preocupações com a cultura e contexto em que as pessoas estão inseridas (SCHMIDT et al., 2005) e aspectos religiosos (PETERSON e WEBB, 2006; MOREIRA-ALMEIDA, LOTUFO NETO e KOENIG, 2006). Percebe-se ainda uma valorização cada vez maior da percepção individual de cada indivíduo sobre sua própria vida na investigação do tema (GILL e FEINSTEIN, 1994) e da qualidade de vida do trabalhador (OLIVEIRA, 1997; LACAZ, 2000; VASCONCELOS, 2001; ASSUNÇÃO, 2003).

Usualmente utilizado no plano político e jornalístico, conforme aborda Farquhar (1995), o tema qualidade de vida é utilizado tanto no cotidiano como em várias áreas de pesquisa como sociologia, medicina, psicologia, economia, ergonomia, geografia, história e filosofia; suas definições são tão numerosas e divergentes quanto os métodos de avaliação. Há de se destacar a abordagem superficial do tema evitando definir exatamente o que pretendem medir ou apresentando o tema de forma limitada. Desta maneira, qualquer estudo com méritos tem que definir claramente seu assunto e então decidir as 
$\overline{\text { direções metodológicas a seguir; talvez por ser um termo ambíguo, que varia de pessoa para pessoa }} \mathrm{e}$ especialmente por ser um tema multidisciplinar suas definições são multifatoriais. As preocupações com qualidade de vida são crescentes em muitos estudos que buscam compreender e sugerir formas para que as pessoas vivam e, além do mais é o que se deseja quando se relaciona as políticas públicas como, por exemplo, os tratamentos de saúde.

Análises da estrutura demográfica e social que podem prever as condições de interação que conduzem à satisfação, bem-estar e qualidade de vida são apresentadas por Ferris (2006). A estrutura demográfica pode ser definida a partir da interação de variáveis como idade e gênero (que define a estrutura demográfica básica) e outras secundárias como etnia, distribuição demográfica, distribuição de renda, acesso à educação e saúde, balanço entre população jovem e idosa e entre pessoas economicamente ativas e inativas. O ambiente e políticas públicas, a personalidade, liberdade, democracia, família, cultura, locais para lazer, dentre outros, completam uma rede de fatores que influenciam na forma com que as pessoas vivem e na sua qualidade de vida.

Algumas questões como a distribuição idade/gênero de uma população possui grande influência na qualidade de vida. Em relações de dependência, por exemplo, quanto mais dependente a pessoa for, normalmente menor será a sua qualidade de vida. As categorias demográficas emprego/desemprego também são elementos fundamentais da qualidade de vida. A instituição econômica deve prever um equilíbrio entre emprego e desemprego, sendo flexível para expandir a geração de emprego conforme a demanda, sendo que o tamanho dessa geração é uma conseqüência da taxa de fertilidade de 20 anos antes. Se a economia não pode se ajustar, outros ajustes serão necessários como o deslocamento de pessoas á procura de empregos em outros locais (FERRIS, 2006).

Outra característica demográfica importante segundo o mesmo autor é o tamanho da população e da comunidade, ou seja, o tamanho demográfico da comunidade afeta o número e variedade de instituições (o número de igrejas, hospitais, estabelecimentos industriais) que possibilitam participação e interação social. A oportunidade para participação é afetada pela presença ou ausência de instituições e, conseqüentemente, pode influenciar na existência de oportunidades de crescimento e satisfação com a vida. Outros fatores demográficos podem influenciar na qualidade de vida como a variação da fertilidade e mortalidade em relação à provisão de comida afetando a pobreza, estrutura familiar, aumento da população, a divisão de trabalho, relações socioeconômicas. A distribuição de bens e serviços dá origem ao poder, riqueza, renda, pobreza, prestígio, acesso ou não a educação. A distribuição de riqueza ou renda 
inclui um marco de desigualdade e uma das características estruturais mais importantes. Além disso, a religião, os dogmas também estão envolvidos com as questões e percepções de qualidade de vida.

\section{SAÚDE}

Saúde e doença configuram processos compreendidos como um continuum, relacionados aos aspectos econômicos, socioculturais, à experiência pessoal e estilos de vida (SEIDL e ZANNON, 2004). A avaliação do estado de saúde está diretamente relacionada à qualidade de vida influenciada pelo gênero, escolaridade, idade, condição econômica e presença de incapacidades (PEREIRA et al., 2006).

A Organização Mundial da Saúde (OMS) conceitua saúde como um estado de completo bem-estar físico, mental e social e não apenas a ausência de doença (WHO, 1946). Segre e Ferraz (1997) questionam e apontam como utópico e bastante subjetivo tal conceito. Os autores abordam que mesmo sendo um avanço para época é preciso ser revisto, pois talvez seja impossível o ser humano atingir uma "situação de perfeito bem-estar físico, mental e social”, já que, termos como perfeição, bem-estar ou felicidade não podem ter conceitos em si mesmos sem avaliar o contexto que lhes empreste sentidos a partir do significado, linguagem e da experiência íntima do sujeito. Uma das falhas da definição, segundo os autores, é fazer destaque entre o físico, o mental e o social, pois até mesmo a chamada medicina psicossomática, encontra-se superada, pela vivência psicanalista que aborda a inexistência de uma clivagem entre mente e soma, sendo o social também inter-agente, de forma nem sempre muito claro, com os dois aspectos mencionados. Acredita-se que este conceito seja algo a perseguir, um ideal e talvez um estado de razoável harmonia entre o sujeito e sua própria realidade seria um conceito de saúde mais adequado.

Embora saúde e qualidade de vida sejam muitas vezes utilizadas como sinônimos são conceitos que apresentam especificidades, mas também uma grande relação. Existem evidências científicas abundantes, conforme é abordado por Buss (2000), que mostram a contribuição da saúde para a qualidade de vida de indivíduos ou populações, além da contribuição de muitos componentes da vida social para uma boa qualidade de vida e para que se alcance um perfil elevado de saúde. O mesmo autor complementa que é necessário mais do que o acesso a serviços médico-assistenciais de qualidade, é preciso enfrentar os determinantes da saúde em toda a sua amplitude, o que requer políticas públicas saudáveis, uma efetiva articulação intersetorial do poder público e a mobilização da população.

Considerando qualidade e condições de vida como sinônimos, o autor ainda aborda que o tema da influência da saúde sobre as condições e a qualidade de vida, e vice-versa. Assim, tem-se questionado o 
papel de áreas como a medicina, saúde pública e, num sentido mais genérico, do setor saúde no enfrentamento do que seriam as causas mais amplas e gerais dos problemas de saúde.

Particularmente em países como o Brasil e outros da América Latina, segundo Buss (2000), a péssima distribuição de renda, o analfabetismo e o baixo grau de escolaridade, assim como as condições precárias de habitação e ambiente têm um papel muito importante nas condições de vida e saúde. Em um amplo estudo sobre as tendências da situação de saúde na Região das Américas recentemente publicado em 1998, a OPAS (Organização Pan-Americana da Saúde) mostra, de forma inequívoca, que os diferenciais econômicos entre os países são determinantes para as variações nas tendências dos indicadores básicos de saúde e desenvolvimento humano. A redução na mortalidade infantil, o incremento na esperança de vida, o acesso à água e ao saneamento básico, o gasto em saúde, a fecundidade global e o incremento na alfabetização de adultos apresentam função direta do Produto Nacional Bruto dos países. Entretanto, demonstrar que a qualidade/condições de vida afeta a saúde e que esta influencia fortemente a qualidade de vida, não é o único desafio. Embora sobejamente demonstradas, restam muitas questões a serem definidas e respondidas neste campo de investigação, principalmente a respeito de formas de ações que possam, mais eficazmente, influenciar de forma favorável à qualidade de vida.

\section{ESTILO DE VIDA}

Diversos hábitos do dia-a-dia podem levar, em conjunto com as características individuais, ao surgimento ou agravamento de fatores de risco relacionados ao acometimento de doenças e aumento da mortalidade. Hábitos como fumar e beber, além de um dos grandes problemas de saúde pública mundial - obesidade, estão relacionados à elevação dos níveis de colesterol, pressão arterial, dentre outros.

Num mundo contemporâneo, dentro de uma estrutura capitalista, na qual, muitas vezes as pessoas necessitam dedicar-se integralmente às atividades profissionais para garantia de subsistência, tem-se percebido um aumento importante de indivíduos acometidos pelas chamadas doenças crônicodegenerativas, que são resultados, dentre outros fatores, de hábitos de vida não saudáveis como o sedentarismo. Investigações realizadas no Brasil analisando, por exemplo, funcionários de um banco, verificaram a prevalência de sedentarismo em $57 \%$ dos indivíduos avaliados, o consumo diário ou semanal de bebidas alcoólicas em $44 \%$, enquanto $30 \%$ eram fumantes e $35 \%$ apresentavam sobrepeso ou obesidade (CHOR, 1997). Esse alarmante quadro, embora relacionado a uma realidade em particular, pode ser considerado como um indicador de outras realidades preocupantes e que merecem melhor atenção. 
Pesquisas em diversos países, inclusive no Brasil, conforme aborda Nahas (2003), têm mostrado que o estilo de vida, mais do que nunca, passou a ser um dos mais importantes determinantes da saúde de indivíduos, grupos e comunidades. Segundo o autor, estilo de vida pode ser considerado como um conjunto de ações habituais que refletem as atitudes, os valores e as oportunidades na vida das pessoas. Desta forma, o estilo de vida ativo passou a ser considerado fundamental na promoção da saúde e redução da mortalidade por todas as causas, já que, para grande parte da população, os maiores riscos para saúde e o bem-estar, tem origem no próprio comportamento individual, resultante tanto da informação e vontade da pessoa, como também das oportunidades e barreiras presentes na realidade social. Existem, assim, fatores positivos e negativos no estilo de vida que comprovadamente afetam a saúde e bem-estar, a curto e/ou longo prazo. Principalmente a partir da meia idade (40-60 anos), a mobilidade, a autonomia e a qualidade de vida das pessoas são variáveis diretamente associadas aos fatores do estilo de vida e aos declínios morfofisiológicos (TEIXEIRA, PEREIRA e ETCHEPARE, 2006; TEIXEIRA; PEREIRA, 2008).

\section{BEM-ESTAR E FELICIDADE}

Os conceitos de felicidade e bem-estar estão intimamente ligados à qualidade de vida e, em muitos casos, os conceitos são inclusive tratados como sinônimos. Dela Coleta e Dela Coleta (2006) destacam três aspectos importantes sobre as dimensões de bem-estar: a) o bem-estar subjetivo nem sempre é afetado por condições materiais, de saúde, conforto e riqueza, sabendo-se que a influência destes aspectos depende dos valores e expectativas do indivíduo, do grupo a que pertence e da sociedade em que vive; $b$ ) medidas de aspectos positivos têm sido utilizadas, a despeito dos aspectos negativos na vida do indivíduo, sabendo-se que o bem-estar subjetivo tem sido considerado como resultado do balanço entre afetos positivos e negativos, sendo ignorado até o momento quando eles se equilibram; c) por último, as medidas de bem-estar subjetivo incluem um julgamento global de todos os aspectos da vida, e embora o afeto ou a satisfação quanto a algum domínio em particular possa ser avaliada, a ênfase é geralmente colocada sobre o julgamento geral da vida da pessoa.

O caráter subjetivo e individual de bem-estar é importante inclusive considerando as relações de saúde. Doenças como o infarto, a úlcera péptica, a colite irritativa, a asma brônquica, e até mesmo o câncer guardam profundos vínculos com os estados afetivos dos sujeitos. Além disso, a escolha do termo sujeito é relevante no sentido que a doença somática é apenas uma via a mais para externar a turbulência afetiva, tendo sido essa via inconscientemente buscada pelo sujeito incapaz de harmonizar os seus conflitos interiores. Neste sentido, há a necessidade de tratar o doente e não apenas a doença (SEGRE e FERRAZ, 1997). 


\section{LIBERDADE, AUTONOMIA E POSSIBILIDADES DE CRESCIMENTO}

A qualidade de vida de acordo com Santin (2002) não pode apenas ser considerada como uma questão epistemológica, mas ética, pois se constrói alicerçada não sobre conhecimentos, mas sobre valores e decisões. O conhecimento do que é bom e saudável para a vida não é suficiente para orientar o viver segundo tal informação científica. Depende de uma decisão, e esta se dá diante de um valor, pois o ser vivo, humano ou não, é um sistema auto-referido autopoioético, o que quer dizer que ele se realiza com autoconstrução e auto-organização. A autopoiese tem duas funções: criar e ordenar os elementos que constituem tal organismo, e criar os elementos que o protegem das agressões. A cultura corporal, segundo esta tese (explorada por Maturana), nada mais seria do que oferecer as condições e os recursos necessários para que essa dupla tarefa possa se realizar com eficiência. E, uma vez proporcionado tal ambiente, acontece o que se entende como qualidade de vida.

A autonomia e principalmente a possibilidade do ser humano desenvolver suas potencialidades parece ser um ponto crucial para a qualidade de vida e isso depende de inúmeros fatores. Conforme a teoria da autopoiese de Maturana, as pessoas são seres individuais e autônomos, mas que geram em sua vida fenômenos gerais semelhantes entre si e, como um sistema auto-referido, opera somente quanto à ação faz sentido em relação a si mesmo (ROMESÍN e GARCÍA, 2002). Assim, a princípio, dentro de algumas barreiras, o ser humano é livre para buscar sua qualidade de vida, no entanto isso tem que fazer sentido, sendo este individual e subjetivo. As barreiras em que se é sujeito para perseguir as aspirações e o sentido/desejo de alcançá-las vão resultar até onde as pessoas podem "crescer".

\section{PERCEPÇÃO}

No estudo sobre qualidade de vida a percepção pessoal de cada um é bastante importante. Ao analisar a qualidade de vida de longe ou apenas de forma observacional informações podem ser perdidas, pois como já foi discutido anteriormente o conceito é por excelência subjetivo e multidimensional. Apesar disso, é importante salientar que essa percepção do mundo em relação à qualidade de vida pode ser destorcida como qualquer outra percepção. Neste caso, o conceito particular de qualidade de vida, o grau de cultura, escolaridade, vivências, recepção de informações, influência da mídia, dentre outros fatores podem influenciar a percepção da qualidade de vida. Mesmo assim, defende-se aqui que a opinião de cada sujeito deve ser priorizada.

A percepção do mundo ou a forma como cada pessoa o percebe é tema de investigação já dos primeiros pensadores na história ocidental. Platão (427-347 a.C.) já tratava em seus trabalhos e ilustrou em uma de 
suas alegorias "O Mito de Caverna" que a percepção que as pessoas têm do mundo são diferentes entre os sujeitos e nem sempre correspondem a realidade, se é que se pode afirmar que existe uma realidade verdadeira.

A percepção comportamental se refere ao ponto de contato entre o mundo físico e o da mente, sendo um processo psicofísico: um estímulo físico excita, por exemplo, os receptores dos olhos, essa excitação é convertida em impulsos elétricos, que percorrem vias nervosas até chegar ao cérebro, os quais provocam uma mudança estrutural (chamada engrama) da área do córtex (que recebe o impulso) - até aqui todo o processo é físico. De alguma maneira o engrama é convertido em uma cópia mental do objeto, chamada experiência, idéia ou representação, que por sua vez é percebida pela consciência (considerada por essas teorias como um órgão interno de percepção) - essa é a parte mental da percepção (LOPES a ABIB, 2002).

\section{CONSIDERAÇÕES FINAIS}

Diante das considerações realizadas pode se perceber um crescente interesse pelo tema qualidade de vida e os grupos de qualidade de vida de organizações como a OMS têm contribuído para a clarificação do tema. Apesar disso, por se tratar de um conceito complexo, abrangente e subjetivo, ainda existem imprecisões de conceitos e muitas variáveis sendo associadas em diferentes investigações. De forma geral a saúde, o estilo de vida e as condições de vida são variáveis diretamente relacionadas à qualidade de vida.

Maiores investigações sobre o tema em diferentes populações e contextos são importantes. A identificação da importância de variáveis associadas em diferentes populações pode colaborar para que os estudos proponham, de forma mais objetiva, formas para que as pessoas consigam melhorar sua qualidade de vida individual, em parceria com os profissionais da saúde e por meio de políticas públicas.

\section{REFERÊNCIAS}

ASSUNÇÃO, A. À. Uma contribuição ao debate sobre as relações saúde e trabalho. Ciência \& Saúde Coletiva, Rio de Janeiro, v. 8, n. 4, p. 1005-1018, 2003.

BUSS, P. M. Promoção da saúde e qualidade de vida. Ciência \& Saúde Coletiva. Rio de Janeiro, v. 5, n. 1, p. 163-177, 2000. 


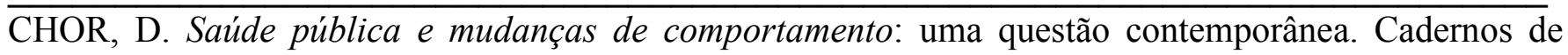
Saúde Pública, Rio de Janeiro, v. 15, n. 2, p. 423-25, 1999.

DANTAS, R. A. S.; SAWADA, N. O.; MALERBO, M. B. Pesquisas sobre qualidade de vida: revisão da produção científica das universidades públicas do estado de São Paulo. Revista Latino-Americana de Enfermagem, Ribeirão Preto, v. 11, n. 4, p. 532-8, 2003.

DELA COLETA, J. A.; DELA COLETA, M. F. Felicidade, bem-estar subjetivo e comportamento acadêmico de estudantes universitários. Psicologia em Estudo, Maringá, v.11, n.3, p. 533-539, 2006.

FARQUHAR, M. Definitions of quality of life: a taxonomy. Joumal of Advanced Nursing, Oxford, v. 22, n.3, p.502-508, 1995.

FERRISS, A. L. A Theory of Social Structure and the Quality of Life. Applied Research in Quality of Life, Columbia, v.1, n. 1, p.117-123, 2006.

FORATTINI, O. P. Qualidade de vida e meio urbano. A cidade de São Paulo, Brasil. Revista de Saúde Pública, São Paulo, v. 25, n.2, p. 75:86, 1991.

GILL, T. M.; FEINSTEIN, A. R. A critical appraisal of the quality of quality-of-life measurements, Journal of the American Medical Association, Chicago, v. 272, n. 8, p. 619-626, 1994.

LACAZ, F. A. C. Qualidade de vida no trabalho e saúde/doença. Ciência \& Saúde Coletiva. Rio de Janeiro, v. 5, n. 1, p. 151-161, 2000.

LOPES, C. E.; ABIB, J. A. D. Teoria da Percepção no Behaviorismo Radical. Psicologia: Teoria e Pesquisa, Brasília, v. 18, n. 2, p. 129-137.

MICHALOS, A. C.; ZUMBO, B. D.; HUBLEY, A. Health and the quality of life. Social Indicators Research, Dordrecht v. 51, n.3: 245-286, 2000.

MOREIRA-ALMEIDA, A.; LOTUFO NETO, F.; KOENIG, H. G. Religiosidade e saúde mental: uma revisão. Revista Brasileira de Psiquiatria, São Paulo, v. 28, n. 3, p.242.250, 2006.

NAHAS, M. V. Atividade física, saúde e qualidade de vida: conceitos e sugestões para um estilo de vida ativo. 3. ed. Londrina: Midiograf, 2003.

OLIVEIRA, S. A qualidade da qualidade: uma perspectiva em saúde do trabalhador. Cadernos de Saúde Pública, Rio de Janeiro, v. 13, n. 4, p. 625-634, 1997.

PEREIRA, R. J.; COTTA, R. M. M.; FRANCESCHINI, S. C. C.; RIBEIRO, R. C. L.; PRIORE, S. E.; CECON, P. R. Contribuição dos domínios físico, social, psicológico e ambiental para a qualidade de vida global de idosos. Revista de Psiquiatria do Rio Grande do Sul, Porto Alegre, v. 28, n. 1, p. 27-38, 2006. 
PETERSON, M.; WEBB, D. Religion and spirituality in quality of life studies. Applied Research in Quality of Life, Columbia, v. 1, n. 1, p. 107-116, 2006.

PIRES, L. P. D. E.; MATIELLO, E. M.; GONÇALVES A. Alguns olhares sobre aplicações do conceito de qualidade de vida em educação fisica/ciências do esporte. Revista Brasileira de Ciências do Esporte, Campinas v. 20, n. 1, p. 53-57, 1998.

PLATÃO. A República. Coleção a abra-prima de cada autor. São Paulo: Martin Claret, 2005.

RENWICK, R.; BROWN, I. The Center for Health Promotion's Conceptual Approach to Quality of Life. In.: RENWICK, R.; BROWN, I.; NAGLER, M. (Eds.) Quality of life in health promotion and rehabilitation: conceptual approaches, issues and applications. Thousand Oaks, California: Sage Publications, 1996. p. 75-86.

ROMESÍN, H. M.; GARCÍA, F. J. Varela. De máquinas e seres vivos: autopoiese - a organização do vivo. 3. ed. Porto Alegre: Artes Médicas, 2002.

SANTIN, S. Cultura corporal e qualidade de vida. Kinesis, Santa Maria, n. 27, p. 116-186, 2002.

SCHMIDT, S.; POWER, M.; BULLINGER, M. The conceptual relationship between health indicators and quality of life: results from the cross-cultural analysis of the EUROHIS field study. Clinical Psychology and Psychotherapy, Hoboken. n. 12, p. 28-49, 2005.

SEGRE, M.; FERRAZ, F. C. O conceito de saúde. Revista de Saúde Pública, São Paulo, v. 31, n. 5, p. 538-542, 1997.

SEILD, E. M. F.; ZANNON, C. M. L. C. Qualidade de vida e saúde: aspectos conceituais e metodológicos. Cadernos de Saúde Pública, Rio de Janeiro, v. 20, n. 2, p. 580-8, 2004.

TANI, G. Esporte, educação e qualidade de vida. In: W. W. Moreira; R. Simões. (Org.). Esporte como fator de qualidade de vida. Piracicaba: UNIMEP, 2002, p. 103-116.

TEIXEIRA, C. S.; PEREIRA, É. F; ETCHEPARE, L. S. O envelhecimento e o sistema músculoesqulético. Lecturas Educación Física y Deportes, Buenos Aires, v. 11, n. 101, 2006.

TEIXEIRA, C. S.; PEREIRA, É. F. Alterações morfofisiológicas associadas ao envelhecimento humano. Lecturas Educación Fisica y Deportes, Revista Digital, Buenos Aires, v. 13, n.124, 2008.

VASCONCELOS, A. F. Qualidade de vida no trabalho: origem, evolução e perspectivas. Cadernos de Pesquisa em Administração, São Paulo, v. 8, n.1, p.23-35, 2001.

WHO (World Health Organization). Constitution of the World Health Organization. Basic Documents. WHO. Genebra, 1946. 
ARTIGO

$\overline{\text { WHOQOL Group. The development of the World Health Organization quality of life assessment }}$ instrument (the WHOQOL). In: ORLEY, J.; KUYKEN, W. (Eds.). Quality of life assessment: international perspectives. Heidelberg: Springer Verlag; 1994. p. 41-60. 
Érico Felden Pereira

Universidade Federal do Paraná

Anderlei dos Santos

Universidade Federal do Paraná

Clarissa Stefani Teixeira

Universidade Federal de Santa Catarina

Lizandra Salau da Rocha

Universidade Federal de Santa Maria

Rosimeide Francisco Santos Legnani

Universidade Federal do Paraná

Eugenio Andrés Díaz Merino

Universidade Federal de Santa Catarina

Referência do artigo

ABNT

PEREIRA. E. F., et al. Qualidade de vida: conceito e variáveis relacionadas. Conexões, v. 7, n. 3, p. 14-28, 2009

APA

Pereira É. F., SANTOS A.;TEIXEIRA C. S.; ROCHA L. S.; LEGNANI R. F. S. \& MERINO. E. A. D. (2009). Qualidade de vida: conceito e variáveis relacionadas. Conexões, 7(3), 14-28.

\section{VANCOUVER}

Pereira EF, SANTOS A, TEIXEIRA CS, ROCHA LS, LEGNANI RFS, MERINO EAD. Qualidade de vida: conceito e variáveis relacionadas. Conexões, 2009; 7(3): 14-28. 